\title{
PÉRDIDAS ECONÓMICAS EN VALOR PRESENTE AL AÑo 2016 CAUSADAS POR EL VULCANISMO EN COSTA RICA ENTRE 1953 Y 2016
}

\author{
ECONOMIC LOSSES IN PRESENT VALUE OF 2016 CAUSED BY THE \\ VOLCANISM IN COSTA RICA FROM 1953 TO 2016
}

\author{
Tatiana Abarca ${ }^{1 *}$ y Guillermo E. Alvarado ${ }^{2,3}$ \\ ${ }^{1}$ Maestría Profesional en Gestión del Riesgo y Atención de Emergencias, \\ Universidad de Costa Rica \\ ${ }^{2}$ Área de Amenazas y Auscultación Sísmica y Volcánica, Instituto \\ Costarricense de Electricidad \\ ${ }^{3}$ Red Sismológica Nacional UCR-ICE \\ * Autora para contacto: tatiana.abarca@gmail.com
}

(Recibido: 04/12/2016; aceptado: 20/03/2017)

\begin{abstract}
An estimate of the economic losses caused by volcanic activity in Costa Rica in the period between 1953 and 2016 in present value is presented. Detailed information of the damage to agriculture, infrastructure is provided, among others, and it is classified according to the year in which the activity occurred and also by the volcano that generated it. During this time there were three national emergencies triggered by volcanic eruptions: Irazú in 1963 - 1965, Arenal in 1968 and Poás in 1990, whose losses were estimated in US\$117.3 million, US\$5,8 million and between US\$1.1 and US\$2.3 million, respectively (american dollars in present value of 2016). If taken into consideration other periods of volcanic crisis, the estimated total economic losses in Costa Rica because of volcanism is between US\$174.1 and US\$193.1 million in present value. This is a minimum amount because many of the economic losses were not historically quantified. Keywords: Volcanism, Rincón de la Vieja, Arenal, Poás, Irazú, Turrialba, economic losses.

RESUMEN: Se presenta una estimación en valor presente de las pérdidas económicas causadas por la actividad volcánica en Costa Rica en el período comprendido entre 1953 y 2016. Se brinda información detallada de los daños en agricultura, ganadería, infraestructura, entre otros, clasificada según el año en que ocurrió la actividad y el volcán que la generó. Durante este tiempo ocurrieron tres emergencias nacionales disparadas por erupciones volcánicas: Irazú en 1963 - 1965, Arenal en 1968 y Poás en 1990, cuyas pérdidas se estimaron (en dólares estadounidenses en valor presente al 2016) en US\$117,3 millones, US\$5,8 millones y entre US\$1,1 y US\$2,3 millones, respectivamente. Si se toman en consideración otros períodos de afectación volcánica, el total estimado de pérdidas económicas en Costa Rica por causa del vulcanismo es de entre US\$174,1 y US\$193,1 millones en valor presente. Este es un monto mínimo, dado que muchas de las pérdidas económicas no fueron histíricamente cuantificadas.

Palabras clave: Vulcanismo, Rincón de la Vieja, Arenal, Poás, Irazú, Turrialba, pérdidas económicas.
\end{abstract}




\section{INTRODUCCIÓN}

En Costa Rica existen cinco volcanes activos históricamente: Rincón de la Vieja, Arenal, Poás, Irazú, y Turrialba, los cuales han presentado actividad eruptiva dentro del período comprendido en este estudio. Su actividad ha provocado pérdidas económicas para el país, particularmente la actividad del volcán Irazú en el período 1963 - 1965, la del volcán Arenal en 1968 y la del volcán Poás en 1990, que fueron declaradas emergencia nacional. Otras pérdidas corresponden con las erupciones de los volcanes Poás entre 1989 y 1994, Rincón de la Vieja en 1991 y 1995, y recientemente el volcán Turrialba entre el 2010 y el 2016 (período evaluado).

El trabajo de Aguilar y Alvarado (2014) realiza una compilación exhaustiva de las pérdidas económicas y en vidas humanas de 1953 al 2005 en precios de cada época. Sin embargo, no se estimó el valor de las pérdidas económicas en valor presente al 2016. Tampoco incorporaron los datos de las pérdidas debido a la actividad eruptiva del volcán Turrialba del 2010 al 2016. Ambas situaciones se subsanan en la presente investigación.

El presente trabajo realiza una comparación de las pérdidas económicas producto de la actividad volcánica en Costa Rica, al estimar el valor de las pérdidas del año en que ocurrieron en valores actuales (tanto en colones costarricenses como su equivalente en dólares estadounidenses), utilizando el Índice de Precios al Consumidor (IPC) y el tipo de cambio promedio de venta del sector público no bancario de diciembre del 2016.

\section{METODOLOGÍA}

Se utilizó como base el trabajo de Aguilar y Alvarado (2014), el cual recopila gran cantidad de datos dispersos en diferentes fuentes de información, y que representa la primera aproximación integrada de los efectos de las erupciones volcánicas para el período 1953 - 2005. En el trabajo en mención se utilizó como fuente primaria la documentación oficial del Gobierno y la de las personas e instituciones directamente involucradas en la evaluación de los daños, así como revistas, publicaciones y periódicos de la época. Principalmente se utilizaron dos informes: uno realizado por la Oficina de Planificación del Gobierno de la República (GCROP, 1964) para la emergencia del volcán Irazú y otro realizado por la Comisión para la Emergencia del Volcán Arenal (CEVA, 1969). Sin embargo, el informe de Irazú cubre desde el inicio de la emergencia hasta agosto de 1964, pero no contempla los 6 meses faltantes de la erupción.

En el presente trabajo, para los datos de pérdidas del período 2006 - 2016, principalmente asociadas con el volcán Turrialba, se compiló información de la Comisión Nacional de Prevención de Riesgos y Atención de Emergencias (CNE), del Ministerio de Agricultura y Ganadería (MAG), del Instituto Costarricense de Electricidad (ICE), del Servicio Nacional de Salud Animal (SENASA), de la Biblioteca Nacional y de diversos artículos publicados por los principales periódicos nacionales. Respecto al reporte de pérdidas en el 2016 se utilizó un informe de acciones operativas elaborado por la $\mathrm{CNE}$, datos suministrados por el ICE e información extraída del periódico La Nación.

A efectos de hacer comparables los valores monetarios que representaron los daños se utilizó el IPC, el cual mide la variación promedio en los precios de un grupo de bienes y servicios que responden a los de mayor importancia dentro del gasto de consumo final de los hogares, según la información publicada en la página web oficial del Instituto Nacional de Estadística y Censos (INEC) (www.inec.go.cr), y por consulta realizada electrónicamente a la Unidad de Índice de Precios de dicha Institución.

Por otra parte, para la conversión de los montos en colones costarricenses a dólares estadounidenses, se utilizó el promedio del tipo de cambio del sector público no bancario de diciembre del 2016, tomado de la página web oficial del Banco Central de Costa Rica (www.bccr.fi.cr).

El ejercicio consiste es determinar la relación entre el IPC del año de interés y el IPC del año 2016, para así ponderarlo por el monto que se requiere obtener en valor presente. Una vez que se tiene este dato, se realiza la conversión a dólares estadounidenses.

Se utilizó el IPC dado que es un instrumento estadístico que permite medir la evolución a 
través del tiempo de los precios de un conjunto de bienes y servicios representativo del consumo final de los hogares. Por lo tanto, permite incorporar el efecto precios de los años transcurridos desde 1953. El índice se actualiza periódicamente dados los cambios que se presentan en el patrón de consumo de los hogares (información que se obtiene de la Encuesta Nacional de Ingresos y Gastos de los Hogares).

\section{PÉRDIDAS ECONÓMICAS EN COSTA RICA CAUSADAS POR EL VULCANISMO}

Costa Rica cuenta con cinco volcanes históricamente activos: Rincón de la Vieja, Arenal, Poás, Irazú y Turrialba (Cuadro 1). Si bien el vulcanismo aporta suelos fértiles, geoturismo, energía geotérmica, entre otras cosas, también provoca efectos negativos para los ciudadanos. Previo a detallar las pérdidas económicas, como resultado de la actividad volcánica en Costa Rica para el período comprendido entre 1953 y 2016, a continuación se realiza una pequeña referencia a cada uno de los volcanes mencionados.

Seguidamente se detallan los datos correspondientes a las pérdidas económicas producto de la actividad volcánica en Costa Rica. Se realiza una clasificación según el año en que ocurrió la actividad y según volcán.

\section{Década 1950 - 1959}

Durante esta década se tiene registro de actividad en el volcán Poás entre 1953 y 1955, con efectos en la agricultura y ganadería, principalmente, debido a la lluvia ácida y a la caída de ceniza. Las pérdidas generadas durante la actividad del volcán Poás durante 1953 fueron estudiadas por Aguilar y Alvarado (2014), quienes se basan en reportes del periódico La Nación. Dichos autores refieren que el área más afectada por la caída de ceniza fue entre Vara Blanca y río el Ángel, así como que los efectos de la ceniza afectaron la ganadería debido a la contaminación del agua y a los pastos con ceniza.
Por su parte, los autores indican que en el transcurso de 1953 las pérdidas variaron entre $\not 25000000$ y $\mathbb{C} 30000000$ debido a la lluvia ácida y ceniza en fincas y terrenos dedicados a la ganadería, según datos obtenidos del periódico La República. Dichos montos se traducen, al día de hoy, en pérdidas entre $\mathbb{C} 15184109706$ y \18 220931 647; o su equivalente en dólares estadounidenses que corresponde a US\$27 393801 y US\$32 872 561, respectivamente.

\section{Década 1960 - 1969}

En el transcurso de la década de 1960 ocurrió la mayor cantidad de actividad volcánica en el país: el Irazú (1963 - 1965), el Rincón de la Vieja (1967) y el Arenal (1968). A continuación se describe la actividad de cada uno de ellos, según el año en que ocurrieron.

\section{Volcán Irazú 1963 - 1965}

La actividad del volcán Irazú inició en 1962 pero fue hasta en marzo de 1963 que se intensificó, cuando la nube de cenizas alcanzó $6,5 \mathrm{~km}$ de altura, provocando gran malestar entre los ciudadanos, razón por la cual se dio la primera declaratoria de emergencia nacional en el país debido a la actividad volcánica (Alvarado, 2009).

La erupción de ceniza se mantuvo por aproximadamente 30 meses, causando daños en la agricultura y la ganadería, entre otros; además de las corrientes laháricas provocadas por las lluvias que destruyeron puentes, la línea de ferrocarril al Caribe, el pueblo de Taras y el abastecimiento de agua potable. Respecto a la actividad del volcán Irazú en este período de tiempo, es importante indicar que el informe realizado por la Oficina de Planificación (GCROP, 1964) recopiló información hasta agosto de 1964, es decir, los últimos seis meses del período de la erupción y los meses posteriores de recuperación económica quedaron por fuera del informe (Alvarado, 2009; Aguilar y Alvarado, 2014).

En relación a las pérdidas en infraestructu$\mathrm{ra}$, se encontraron divergencias en la información referente a la cantidad de casas destruidas 
Cuadro 1

Volcanes activos en Costa Rica.

\begin{tabular}{|c|c|c|c|}
\hline Volcán & Tipo de volcán & $\begin{array}{l}\text { Elevación } \\
\text { (m.s.n.m.) }\end{array}$ & Descripción de la actividad \\
\hline Rincón de la Vieja & Estratovolcán complejo & 1916 & Erupciones freáticas y freatomagmáticas, y lahares. \\
\hline Arenal & Estratovolcán cónico adosado & 1750 & $\begin{array}{l}\text { Erupciones peleanas, vulcanianas, estrombolianas, } \\
\text { efusión de lava, flujos piroclásticos y emisión de gases. } \\
\text { Entró en reposo a partir del } 2010 .\end{array}$ \\
\hline Poás & Estratovolcán complejo & 2708 & Erupciones freáticas y lluvia ácida. \\
\hline Irazú & Escudo volcánico complejo & 3432 & Actividad estromboliana, freatomagmática y lahares. \\
\hline Turrialba & Estratovolcán complejo & 3340 & Erupciones freatomagmáticas y lluvia ácida. \\
\hline
\end{tabular}

y afectadas por los lahares en Taras de Cartago, según el siguiente detalle: en un informe del ICE (1965) se menciona un total de 300 casas destruidas; Krushensky (1972) se refiere a unas 400 casas afectadas; Mora (1993) indica que las casas destruidas fueron 575; mientras que Young et al. (1998) menciona que fueron 350.

En el informe de la Oficina de Planificación (GCROP, 1964) se establece que las pérdidas económicas por destrucción de viviendas fueron por $\mathbb{C} 5596$ 312, cifra muy similar a la presentada por el ICE (1965) (\$5 600 000). Dichos montos corresponden en valor presente a $\mathbb{2} 646832699$ y \2 648576 976, que en dólares estadounidenses equivalen a US\$4 905903 y US\$4 909 136, respectivamente.

Las pérdidas en agricultura se clasifican según el siguiente detalle: café, tabaco, papa y varios cultivos. Referente al café, se indica que en las provincias de San José, Alajuela y Heredia, la zona afectada fue de 14700 hectáreas de café, y que la caída de ceniza y la sequía ocasionaron tres plagas, causando daños a las plantaciones.

Según diferentes autores, las pérdidas en las plantaciones de café producto de la actividad del Irazú para el período en estudio fueron las siguientes: $\mathbb{C} 112852142$ (GCROP, 1964), \135 700000 (ICE, 1965), \9 814000 (MAG, 1963 ) y $\mathbb{2} 26600000$ (dato en que coinciden los autores Armbrister, 1964 y Parsons, 1967).

Por su parte, para el caso del tabaco y la papa se cuenta con datos del informe de la Oficina de Planificación (GCROP, 1964) y del ICE (1965), siendo que los principales daños se registraron en la zona tabacalera de Puriscal, mientras que para el caso del cultivo de papa los principales lugares afectados fueron Llano Grande, Tierra Blanca, San Juan de Chicuá y Coliblanco.

Las pérdidas en el cultivo del tabaco ascienden a $\mathbb{C} 873789$ y $\not \subset 900$ 000, mientras que en el cultivo de papa fueron por $\mathbb{C} 1102000$ y $\mathbb{C} 1100$ 000, según el informe de la Oficina de Planificación (GCROP, 1964) y del ICE (1965), respectivamente.

En el siguiente cuadro (Cuadro 2) se presentan los datos de pérdidas en cultivos, según lo descrito previamente, en dólares estadounidenses, una vez que los datos fueron actualizados a valor presente (para este último detalle, ver el Anexo 1).

El cuadro anterior muestra las diferencias en las valoraciones para un mismo cultivo según el autor. Aguilar y Alvarado (2014) indican que las diferencias pueden deberse a los años en que los autores realizaron el estudio, así como las zonas que hayan estudiado y los datos que hayan considerado como pérdidas.

Por otro lado, las pérdidas en la ganadería se refieren, principalmente, a la muerte de animales y a abortos de vacas que se encontraban en períodos avanzados de gestación. Adicionalmente, se registraron pérdidas por la caída de ceniza en zonas tales como San Isidro de Coronado, parte del cantón de Goicoechea, parte de Tres Ríos, Turrialba y en la zona norte de Heredia. Como medidas preventivas, se trasladaron 560 cabezas de ganado a zonas libres de ceniza en Cartago, Coronado y Turrialba, además, se exportaron 116 animales a Panamá y 60 a Honduras (Aguilar y Alvarado, 2014). 
Cuadro 2

Pérdidas en cultivos por la actividad del Irazú (1963 - 1965) en valor presente al 2016 en dólares estadounidenses.

\begin{tabular}{ccccc}
\hline Café & Tabaco & Papa & Varios cultivos & Referencia \\
\hline US\$96 293 580 & US\$745 580 & US\$940 306 & - & GCROP (1964) \\
US\$115 789 020 & US\$767945 & US\$938599 & - & ICE (1965) \\
US\$8 374 012 & - & - & US\$1 134 852 & Ambrister (1964) \\
US\$22 697037 & - & - & - & Parsons (1967) \\
US\$22 697037 & - & - &
\end{tabular}

Las pérdidas se clasifican según el siguiente detalle: por improductividad $\not 1869781$ (MAG, 1963), por afectación de ceniza $\mathbb{C} 1350000$ (GCROP, 1964), por menor producción lechera $\mathbb{C} 961200$ (GCROP, 1964) y un detalle no especificado de $\mathbb{C} 1400000$ por parte del ICE (1965).

En el cuadro 3 se presentan los datos de pérdidas en la ganadería en su monto equivalente en dólares estadounidenses, una vez que los datos fueron actualizados a valor presente (para este último detalle, ver el Anexo 2).

A pesar de que en el cuadro anterior, se menciona como parte de las pérdidas en ganadería aquella proveniente de menor producción lechera, Aguilar y Alvarado (2014) indican otros montos adicionales, relacionados con pérdidas por producción lechera, según el siguiente detalle: $\mathbb{C} 7920000$ del informe GCROP (1964) y $\mathbb{C} 7900000$ del informe del ICE (1965). Estos montos corresponden en valor presente a $\mathbb{C} 3745844580$ y $\mathbb{\$} 3736385377$, que en dólares estadounidenses equivalen a US\$6 757915 y US\$6 740 849, respectivamente.

En cuanto a las pérdidas en infraestructura, las mismas se deben al lahar del río Reventado que afectó el poblado de Taras, siendo así que el informe de la Oficina de Planificación (GCROP, 1964) menciona pérdidas por un total de $\mathbb{C} 6652$ 296, monto que coincide con el total de $\mathbb{C 6} 700000$ del informe del ICE (1965). En valor presente, dichos montos corresponden a $\mathbb{C 3} 146271075$ (US\$5 676218 ) y $\mathbb{~} 3168833$ 168 (US\$5 716 923), respectivamente.

Se estiman como pérdidas indirectas aquellas provenientes de la devaluación de tierras y de las afectaciones en los servicios públicos, siendo que para el primer caso el informe de la Oficina de Planificación (GCROP, 1964) estimó los daños en $\mathbb{C} 100000000$ y el informe del ICE (1965) en $\mathbb{C} 100400$ 000, cuyos equivalentes en dólares estadounidenses corresponden a US\$85 327207 y a US\$85 668516 (para ver el detalle de estos montos en valor presente, ver el Anexo 3).

Adicional a las pérdidas previamente mencionadas, Aguilar y Alvarado (2014) hacen un recuento de las inversiones que requirió el país para la recuperación, basándose en la información del GCROP (1964), según se presenta en el cuadro 4 (para ver el detalle de los montos en valor presente, ver Anexo 4).

A manera de ejemplo, se hace referencia a algunas de las categorías a las cuales se destinaron

Cuadro 3

Pérdidas en ganadería por la actividad del Irazú (1963 - 1965) en valor presente al 2016 en dólares estadounidenses.

\begin{tabular}{ccc}
\hline Ganado sacrificado por & Total & Referencia \\
\hline Afectación por ceniza & US\$1 151 917 & GCROP \\
Menor producción lechera & US\$820 165 & GCROP \\
Improductividad & US\$742 160 & MAG \\
No especificado & US\$1 194 581 & ICE \\
\hline
\end{tabular}


recursos para la recuperación. En primer lugar, dentro de los pastos se tomó en consideración la cantidad de hectáreas destinadas al pastoreo que fueron afectadas por la ceniza, así como la necesidad de semillas, siembra y reparación de cercas y pajas de agua.

Se destinó un fondo para la construcción en la zona baja del Reventado y en distintas zonas de dicha cuenca, a la compra de equipo de construcción, y a operaciones de socorro de emergencia, entre otros. Por su parte, la municipalidad de San José debió cambiar sus sistemas de limpieza, recolección y tratamiento de residuos de las calles para crear un nuevo sistema para limpiar las cenizas, lo que requirió invertir en equipo de trabajo.

Aguilar y Alvarado (2014) indican que para atender esta emergencia se recibieron donaciones de países tales como Nicaragua, Venezuela y Estados Unidos; así como de instituciones tales como el MAG, el Instituto Nacional de Seguros (INS) y la Caja Costarricense del Seguro Social (CCSS).

\section{Volcán Rincón de la Vieja (1967)}

Aguilar y Alvarado (2014) se basan en reportes del periódico La República para obtener los registros de pérdidas de la actividad de 1967 del volcán Rincón de la Vieja, en donde dichas pérdidas se estimaron entre $\mathbb{C} 20000000$ y $\mathbb{C} 35000000$. En valor presente, estos montos representan $\not 4079545455$ (US\$16 380497 ) y $\not \subset 15889204545$ (US\$28 665 869), respectivamente.

\section{Volcán Arenal (1968)}

En 1968 ocurrió la explosión del volcán, de una manera violenta, que se extendió del 29 al 31 julio. Se menciona que esta erupción es la más importante en el país en lo que se refiere a pérdidas en vidas humanas, a pesar de que el número oficial de muertos y desaparecidos nunca se ofreció, aunque se toma el número de 78 muertes como el más correcto (Alvarado, 2009; Aguilar y Alvarado, 2014).

Unas 339 familias perdieron todo. De ellas, 175 eran procedentes de la provincia de Alajuela
Cuadro 4

Inversiones para la recuperación en valor presente al 2016 en dólares estadounidenses.

\begin{tabular}{|c|c|}
\hline Programa & Costo \\
\hline Pastos & US\$11 049873 \\
\hline Atención al ganado & US\$4 656805 \\
\hline Investigación de suelos & US\$27000 \\
\hline Combate plagas del café & US\$4 266360 \\
\hline Fondo para la construcción & US\$2 000000 \\
\hline Diques y dragados & US\$11 801602 \\
\hline Infraestructura vial & US\$7 278411 \\
\hline Limpieza de calles & US\$1 552014 \\
\hline Agua potable & US\$1 761154 \\
\hline Vivienda & US\$12 218429 \\
\hline $\begin{array}{l}\text { Financiamiento a cafe- } \\
\text { taleros }\end{array}$ & US\$17 065441 \\
\hline Industrias en Taras & US\$4 266360 \\
\hline Carreteras & US\$7 193084 \\
\hline $\begin{array}{c}\text { Hospital San Juan de Dios } \\
\text { debido a la ceniza }\end{array}$ & US\$912 506 \\
\hline Total & US\$86049040 \\
\hline
\end{tabular}

y las restantes 164 familias eran de la provincia de Guanacaste. También se menciona que la CEVA -con sus recursos- encargó a la Dirección General de Defensa Civil la reconstrucción y reparación de las viviendas.

Los poblados de Tabacón y Pueblo Nuevo fueron destruidos (para un área total de destrucción de $12-15 \mathrm{~km}^{2}$ ); mientras que los cantones de Tilarán y San Carlos tuvieron pérdidas en agricultura y ganadería. Adicionalmente, los siguientes poblados también sufrieron los efectos de la caída de ceniza, aunque en menor grado debido a la distancia de la población respecto al volcán: San Juan de Guatuso, La Cabaña, Pejibaye, Liberia, Tilarán, La Fortuna, La Leona, San Pedro de Arenal, Arenal, Sangregado, Silencio, e inclusive Managua y Granada (Nicaragua).

En el cuadro 5 se indican los montos de las pérdidas según el siguiente detalle: el monto original publicado por el estudio de la CEVA (1969), el monto equivalente en colones actuales, y su equivalente en dólares estadounidenses. 
Cuadro 5

Pérdidas por la actividad del volcán Arenal (1968) en valor presente al 2016.

\begin{tabular}{cccc}
\hline Pérdidas & Monto (CEVA) & Monto en valor presente & Monto en dólares americanos \\
\hline Daños en fincas & $\mathbb{C} 4995547$ & $\mathbb{C} 2221120316$ & US\$4 007 145 \\
Ganadería & $\mathbb{C} 1026616$ & $\mathbb{C} 456454049$ & US\$823 493 \\
Productos de origen animal & $\mathbb{1} 19479$ & $\mathbb{C} 8660754$ & US\$15 625 \\
Agricultura & $\mathbb{C} 1234754$ & $\mathbb{C} 548996375$ & US\$990 450 \\
\hline Total & $\mathbb{C} 7276396$ & $\mathbb{C} 3235231494$ & US\$5 836 713 \\
\hline
\end{tabular}

El estudio realizado por la CEVA (1969) determinó que las fincas en las zonas de Tronadora, Centro de Tilarán, Quebrada Grande, Santa Rosa, Tierras Morenas, Líbano y La Fortuna presentaban daños en pastos, casas, edificios, entre otros. En cuanto a la agricultura, los principales cultivos afectados fueron los de café, arroz, frijoles, maíz y caña de azúcar. Por su parte, las pérdidas en la ganadería se deben a que gran cantidad de ganado (vacuno, porcino, caballar) tuvo que ser sacrificado. Asimismo, las pérdidas en productos de origen animal se deben a la afectación en la producción de leche y queso.

$\mathrm{Al}$ igual que ocurrió para atender la emergencia del volcán Irazú en 1963 - 1965, en esta emergencia se recibieron donaciones, según el detalle mostrado en el cuadro 6.

\section{Volcán Poás 1989 - 1994}

Durante estos años, la actividad del volcán Poás se concentró en dos períodos específicos: 1989 - 1990 con columnas de vapor y gases sulfurados y clorurados; y en 1994 con actividad exhalativa y freática. La actividad de 1989 - 1990 generó lluvia ácida en los cantones de Poás, Grecia, Valverde Vega y Naranjo, provocando daños en la salud y en edificios públicos y viviendas; adicionalmente, con el cierre del parque nacional Volcán Poás ocurrieron pérdidas en el turismo e industria local, las cuales no fueron cuantificadas (Aguilar y Alvarado, 2014).

Aguilar y Alvarado (2014), con base a información del periódico La Prensa Libre, indican que la lluvia ácida afectó 8 173,65 hectáreas con cultivos de café y fresas, estimando que las pérdidas para los productores de café fueron entre $\$ 75000000$ y $\mathbb{C} 80000000$. En valor presente dichos montos corresponden a $\mathbb{C} 1211837973$ (US\$2 186289 ) y $\mathbb{C} 1292627172$ (US\$2 332 041), respectivamente. No obstante, los autores señalan que el periódico La República publicó que las pérdidas ascendían $\mathbb{C} 38000$ 000, lo cual equivale en valor presente a \613 997907 (US\$1 107 720). Referente a la actividad de 1994, se estimaron pérdidas de 500 hectáreas de café, cinco hectáreas de tomates, tres hectáreas de frijoles, cebolla, repollo, patatas y brócoli, y 460 hectáreas de tierras de pastoreo; sin embargo, no se tienen los montos que representaron dichas pérdidas (Aguilar y Alvarado, 2014).

\section{Volcán Rincón de la Vieja (1991 y 1995)}

Los efectos de la erupción de 1991 se reflejan en los daños en los puentes del río Pénjamo y del río Azul, los cuales equivalen a $\mathbb{C} 2000000$ y $\mathbb{C} 1000000$, respectivamente. En valor presente, estos montos representan $\mathbb{C} 22714273$ (US\$40 979) у $\not 11357137$ (US\$20 490), respectivamente.

Para la erupción de 1995 no se tiene cuantificación de los daños; sin embargo, se tiene registro de un lahar que pasó y arrasó el puente del río Pénjamo (Aguilar y Alvarado, 2014).

\section{Siglo XXI}

\section{Volcán Arenal (2000)}

En agosto del 2000 un flujo piroclástico provocó la muerte de dos personas. Como efecto de 
Cuadro 6

Donaciones para la atención de la emergencia del volcán Arenal en 1968 (basados en Aguilar y Alvarado, 2014) modificados en valor presente al 2016.

\begin{tabular}{cccc}
\hline Donaciones & Monto & Monto en valor presente & Monto en dólares americanos \\
\hline $\begin{array}{c}\text { Entregadas por empresas } \\
\text { públicas y privadas }\end{array}$ & $\mathbb{C} 61000$ & $\mathbb{C} 27121823$ & US\$48 931 \\
$\begin{array}{c}\text { Recibidas de la población } \\
\text { Recibidas del exterior }\end{array}$ & $\mathbb{C} 135492$ & $\mathbb{C} 60242459$ & US\$108 684 \\
\hline Total & $\mathbb{C} 355000$ & $\mathbb{\bigotimes} 157840115$ & US\$284 761 \\
\hline
\end{tabular}

la actividad del volcán en este período, únicamente se tiene registro de que 600 personas fueron evacuadas de los hoteles de la zona, pero no se tiene registro de los costos de dicha evacuación ni de los seguros de las personas afectadas.

\section{Volcán Poás (2005)}

Producto de la actividad del 2005 la población cercana al volcán sintió algunos efectos en su salud, tales como problemas respiratorios e irritación de ojos. El sector suroeste del parque nacional fue severamente afectado; se reportaron pérdidas por aproximadamente $\mathbb{C} 70000000$, lo cual equivale al 2016 a $\mathbb{C} 126992163$ y a US\$229 108.

\section{Volcán Turrialba (2007 - 2016)}

No se cuenta con datos de las pérdidas económicas por la lluvia ácida producto de la actividad exhalativa del volcán Turrialba para el período comprendido entre el 2007 y el 2009. No obstante, se desprende que las principales afectaciones por la actividad del 2007 fueron en pastizales, algunos cultivos e infraestructura de La Picada y Silvia. Por su parte, en el 2008 y 2009 aumentó la emanación de gases, provocando serios efectos en los pastos y en cultivos de papa y zanahoria.

Para la actividad del 2010, siendo que el volcán tuvo su primera erupción en enero, tampoco se cuenta con la cuantificación económica de los daños; sin embargo, los principales efectos producto de la caída de ceniza ocurrieron en la agricultura y en la ganadería, principalmente en los cantones de Alvarado, Oreamuno y Turrialba, siendo la inversión inmediata para la recuperación asumida por los propios afectados.

Entre otros efectos en el 2010, se mencionan daños en aproximadamente 65 fincas con 650 animales (en el cantón de Alvarado), nueve fincas con una población animal de 713 bovinos (en las zonas de La Esperanza, El Retiro, Miravalles, Central, Silvia y La Picada) y 900 bovinos del cantón de Turrialba, y suspensión de clases en cinco cantones de la provincia de Cartago, afectando a 336 estudiantes.

Del 2011 al 2013, el volcán Turrialba efectuó cuatro erupciones importantes, provocando nuevamente efectos sobre la infraestructura y la vegetación, principalmente como consecuencia de los gases volcánicos, la lluvia ácida y la caída de ceniza. En primer lugar, la calidad y cantidad de tóxicos afecta el bosque y produce daños en pastos y cultivos; y en segundo lugar, afecta la vegetación. También es importante rescatar que se han producido impactos en el turismo nacional e internacional, que visita el parque nacional en el cual se encuentra el volcán, dado el cierre para las visitas del público.

En marzo del 2015 el volcán volvió a presentar actividad significativa con altas concentraciones de ceniza que llegaron inclusive a Heredia, Santa Ana y Alajuela. Entre el 12 y el 13 de dicho mes ocurrieron siete fuertes erupciones de ceniza, siendo que en dos de ellas la altura de la pluma de cenizas sobre la cima del volcán alcanzó los $1500 \mathrm{~m}$. Posteriormente, en octubre ocurrieron aproximadamente 116 erupciones, en donde en una de ellas la columna de gas y ceniza se extendió por más de $1000 \mathrm{~m}$ de altura y se 
desplazó a diferentes zonas del Valle Central tales como Rancho Redondo, Moravia, Coronado, Tibás y Guadalupe (Alvarado et al., 2016).

En cuanto a la cuantificación de pérdidas, el único monto del que se tiene conocimiento es que el Consejo Nacional de Vialidad (CONAVI) invirtió cerca de $₫ 610000000$ en las rutas consideradas de evacuación en caso de que ocurriera una emergencia mayor, siendo el monto equivalente en dólares estadounidenses de US\$1 100507.

Por otra parte, a octubre del 2015 se evacuaron 29 animales que estaban presentando, entre otras cosas, problemas respiratorios debido a la inhalación de gases y cenizas. Además, fue mejor evacuarlos dado que la ceniza provoca efectos en los pastos y en las fuentes de agua, por lo que no eran aptos para su consumo.

Otro de los efectos de la caída de ceniza fue la suspensión de vuelos tanto nacionales como internacionales en los dos aeropuertos de la Gran Área Metropolitana, aunque se carece de una cuantificación económica (Alvarado et al., 2016).

En lo que corresponde al 2016, se tomaron los datos del informe de acciones de la actividad volcánica elaborado por la CNE (2016) y que comprende el período del 28 de abril al 26 de mayo. Los principales efectos se deben a la caída de cenizas en la agricultura y la ganadería, que si bien no se cuenta con los montos correspondientes a las pérdidas, se tiene conocimiento de la afectación en los cultivos de repollo, brócoli, coliflor, papa, zanahoria y cebollín; y en 20 fincas $y$ en el total de 1961 animales en los cantones de Alvarado, Oreamuno y Turrialba.

Durante este mes de actividad se contabiliza una inversión de $\mathbb{C} 238401$ 448, según se desglosa en el en el cuadro 7 que contiene el monto en colones así como su equivalente en dólares estadounidenses.

Adicionalmente, se tiene que como producto en la intervención en rutas de evacuación se requirió una inversión de $\mathbb{C} 1570006$ 400, cuya equivalencia en dólares estadounidenses es de US\$2 832464.

Para el período comprendido de abril a junio, el ICE determinó como costo adicional para la atención de eventos asociados a la actividad del volcán un monto total de $\not \subset 15140$ 200, según el siguiente detalle: horas hombre ordinarias \9 383 500; horas extra $\mathbb{C 3} 356700$; y viáticos y combustible $\mathbb{C} 2400000$. Esto representa en dólares estadounidenses un total de US\$27 315,

Cuadro 7

Inversión realizada en emergencia durante parte del 2016 (CNE, 2016).

\begin{tabular}{ccc}
\hline Suministros/Servicios & Monto en $\not \mathbf{~}$ & Monto en US\$ \\
\hline Pulpa deshidratada & 7920000 & 14289 \\
Pacas trasvala & 23952500 & 43213 \\
Horas sobrevuelo & 1086000 & 1959 \\
Horas maquinaria (vagoneta) & 4276950 & 7716 \\
Horas maquinaria (back hoe) & 3298950 & 5952 \\
Sopladoras & 36700000 & 66211 \\
Insumos agrícolas & 156336968 & 282049 \\
Créditos alimentación & 400000 & 722 \\
Créditos combustible & 100000000 & 180 \\
Contrato de cabañas & 434400000 & 784 \\
Horas caminón cisterna & 1500000 & 2706 \\
Aceite mineral & 1092500 & 1971 \\
Broncodilatadores & 1303180 & 2351 \\
\hline Total & 238401448 & 430102 \\
\hline
\end{tabular}


desglosado de la siguiente forma: US\$16 929 en horas hombre ordinarias; US\$6 056 en horas extra; y US\$4 330 en viáticos y combustible.

Adicional a lo anterior, el ICE realizó una inversión en cambio de transformadores por un monto de $\mathbb{C} 8050000$, lo cual representa en dólares estadounidenses US\$14 523.

Según información del periódico La Nación (23 de febrero, 2017) al cierre del 2016 el MAG reportó las pérdidas en los cultivos hortícolas por el orden de $\not{5} 570200000000$, y en el sector ganadero por $\mathbb{2 7 9} 610000000$; lo cual equivale en dólares estadounidenses a US\$1 028703 y US\$504 447. Al respecto, se indica que la ganadería de leche presentó una reducción de entre el $5 \%$ y el $15 \%$ en la producción, siendo que la afectación se presentó en 4226 vacas en producción en fincas en los cantones de Coronado, Moravia, Alvarado, Turrialba y Oreamuno; por su parte, se afectaron 406 hectáreas de cultivos, según se visualiza en el cuadro 8.

En síntesis, las pérdidas económicas del volcán Turrialba ascienden a un monto mínimo de US\$5 938061 .

Algunos ejemplos de dichas pérdidas, producto de la actividad del volcán Turrialba en el transcurso del 2016, se observan en la figura 1.

Cuadro 8

Pérdidas en cultivos

\begin{tabular}{ccc}
\hline Producto & Monto en $\mathbb{C}$ & Monto en US\$ \\
\hline Papa & 243100000 & 439579 \\
Cebolla & 125000000 & 225514 \\
Fresa & 56000000 & 101030 \\
Repollo & 51100000 & 92190 \\
Coliflor & 42000000 & 75773 \\
Brócoli & 38400000 & 69278 \\
Zanahoria & 14600000 & 26340 \\
\hline Total & 570200000 & 1028703 \\
\hline
\end{tabular}

\section{CONCLUSIONES}

La información relacionada con las pérdidas económicas por efectos del vulcanismo se encuentra sumamente segregada, por lo cual es de difícil acceso y se requiere consultar diferentes fuentes, con el agravante que al comparar datos registrados por diversas instituciones para la misma época, se encuentran diferencias significativas en la información.

De manera general, se observa que los principales efectos de la actividad volcánica se concentran en la agricultura, la ganadería y la infraestructura, sin dejar de lado otros efectos tales como daños en los servicios públicos, el comercio, el turismo, y más recientemente el cierre temporal del tráfico aéreo.

La emergencia por la actividad del volcán Irazú en 1963 - 1965 es la que registra mayores pérdidas económicas en el país, tomando en consideración los datos del informe oficial elaborado por la Oficina de Planificación y al obtener los datos de las pérdidas de aquel momento en valor presente, para un total de US\$118,1 millones.

En el Cuadro 9 se muestra un resumen con las pérdidas para cada una de las épocas de actividad volcánica descritas en este trabajo, siendo que para el caso de las emergencias del Irazú y del Arenal en 1963 - 1965 y 1968, respectivamente, únicamente se indican los datos obtenidos de los informes oficiales del Gobierno.

Las pérdidas económicas mínimas para el período comprendido entre 1953 y 2016 estuvieron en el orden entre US\$174,1 y US\$193,1 millones (en dólares estadounidenses y en valor presente), un monto relativamente bajo para un período de más de 60 años. Lo anterior se debe, entre otras razones, a que mucha información no se registró en el momento en que ocurrieron los hechos. Aún para actividad volcánica reciente, las instancias correspondientes no cuentan con la información sobre los montos de las pérdidas. Por ejemplo, no se han evaluado las pérdidas por el cierre del tráfico aéreo debido a la actividad del volcán Turrialba, lo cual tuvo un impacto por la cantidad 

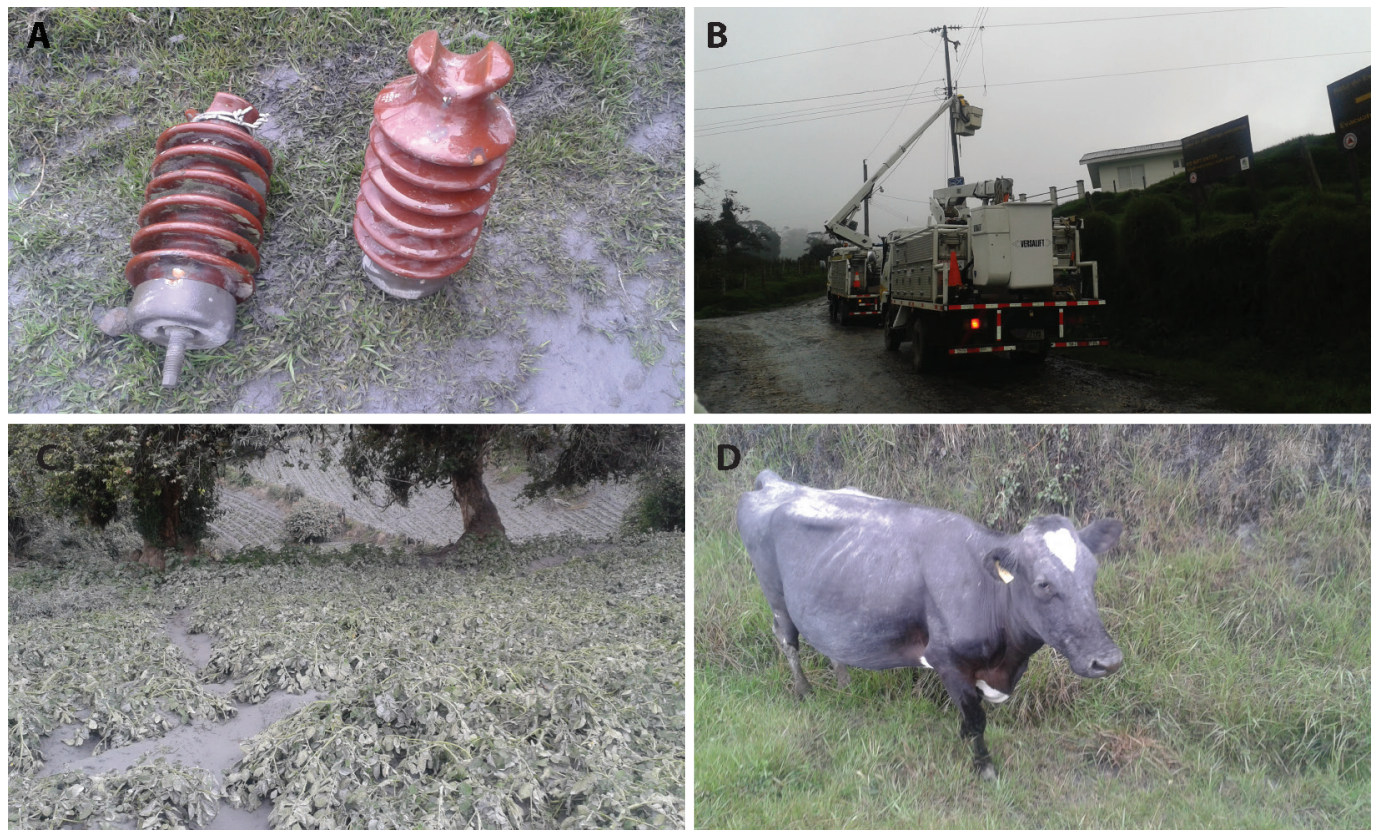

Fig. 1: Ejemplos de pérdidas ocasionadas por la actividad de cenizas del volcán Turrialba en mayo del 2016: a) Aisladores de porcelana dañados, b) Limpieza de transformadores y aisladores, c) Pérdidas de los cultivos, d) Ganado de leche con ceniza.

Cuadro 9

Resumen pérdidas económicas por actividad volcánica en valor presente al 2016 en millones de dólares estadounidenses.

\begin{tabular}{|c|c|c|c|c|c|c|c|c|}
\hline & $\begin{array}{c}\text { Poás } \\
1953-1959\end{array}$ & $\begin{array}{c}\text { Irazú } \\
1963-1965\end{array}$ & $\begin{array}{c}\text { Rincón de la } \\
\text { Vieja } \\
1967 \\
\end{array}$ & $\begin{array}{c}\text { Arenal } \\
1968\end{array}$ & $\begin{array}{c}\text { Poás1 } \\
989 \text { - } 1994\end{array}$ & $\begin{array}{c}\text { Rincón de la } \\
\text { Vieja } \\
1991\end{array}$ & $\begin{array}{l}\text { Poás } \\
2005\end{array}$ & $\begin{array}{c}\text { Turrialba } \\
2015 \text { - } 2016\end{array}$ \\
\hline Agricultura & - & US $\$ 98,0$ & - & US\$1,0 & - & - & - & US\$1,0 \\
\hline $\begin{array}{l}\text { Daños en } \\
\text { fincas }\end{array}$ & - & - & - & US $\$ 4,0$ & - & - & - & - \\
\hline $\begin{array}{l}\text { Destrucción } \\
\text { de viviendas }\end{array}$ & - & US\$4,9 & - & - & - & - & - & - \\
\hline Ganadería & - & US\$8,7 & - & US\$ $\$, 8$ & - & - & - & US\$0,5 \\
\hline Infraestructura & - & US\$5,7 & - & - & - & - & - & US\$3,9 \\
\hline $\begin{array}{c}\text { Productos } \\
\text { de origen } \\
\text { animal }\end{array}$ & - & - & - & US\$0,02 & - & - & - & - \\
\hline Otros & - & - & - & - & - & - & - & US\$0,5 \\
\hline Total & $\begin{array}{c}\text { Entre } \\
\text { US\$27,4 y } \\
\text { US } \$ 32,9\end{array}$ & US\$117,3 & $\begin{array}{c}\text { Entre } \\
\text { US\$16,4 y } \\
\text { US\$28,7 }\end{array}$ & US\$5,8 & $\begin{array}{c}\text { Entre } \\
\text { US\$1,1 y } \\
\text { US\$2,3 }\end{array}$ & $\begin{array}{c}\text { Entre } \\
\text { US\$0,02 y } \\
\text { US\$0,04 }\end{array}$ & $\mathrm{US} \$ 0,2$ & US\$5,9 \\
\hline
\end{tabular}


de vuelos cancelados, implicando para las compañías aéreas costos importantes que debieron haber sido registrados. Tampoco se tiene una idea de las pérdidas en la ganadería en los primeros años, terrenos afectados y sus cultivos, o la movilización de personal civil (policía, personeros de otras instituciones, etc.). Por ello, este costo de US\$193,1 millones es mínimo y quizá el monto real pueda ser el doble o triple puesto que muchos rubros no fueron considerados. Así, resulta necesario que las instituciones establezcan un protocolo de evaluación de pérdidas económicas por desastres, en el presente caso particular, por vulcanismo.

\section{REFERENCIAS BIBLIOGRÁFICAS}

Aguilar, I. y Alvarado, G. E. (2014). Pérdidas humanas y económicas causadas por el vulcanismo en Costa Rica entre 1953 y 2005. Revista Geológica de América Central, 51, 93-128.

Alvarado, G. E. (2009). Los volcanes de Costa Rica: Geología, historia, riqueza natural y su gente ( $3^{\text {ra }}$ ed.). San José: EUNED.

Alvarado, G. E., Brenes-André, J., Barrantes, M., Vega, E., De-Moor, M., Avard, G. ... Carapezza, M. L. (2016). La actividad explosiva del volcán Turrialba (Costa Rica) en el período 2010-2016. Revista Geológica de América Central, 55, 7-60.

Armbrister, T. (1964). The sky is falling. A volcano blows its top, smothering a nation with ash. The Saturday Evening Post, 14, 20-25.

Comisión Nacional de Prevención del Riesgo y Atención de Emergencias (CNE). (2016). Informe de acciones operativas. Actividad Volcánica Volcán Turrialba del 28 de abril al 26 de mayo 2016. San José: CNE. Inf. Interno.
Comisión para la emergencia del volcán Arenal (CEVA). (1969). Comisión para la emergencia del volcán Arenal, Costa Rica. San José: Dpto Audio-Visual Ministerio de Salubridad Pública.

Gobierno de Costa Rica, Oficina de Planificación (GCROP). (1964). Informe: Consecuencias económicas y sociales de la actividad del volcán Irazú. San José: Gobierno de Costa Rica Oficina de Planificación (GCROP)

Instituto Costarricense de Electricidad (ICE). (1965). Informe sobre el problema del río Reventado. San José: ICE. Informe interno.

Krushensky, R. B. D. (1972). Geology of the Istarú quadrangle, Costa Rica. Geological Survey Bulletin, 1358, 1-46.

Ministerio de Agricultura y Ganadería (MAG). (1963). Informe anual de labores. San José: MAG. Informe interno.

Mora, S. (1993). Análisis preliminar de la vulnerabilidad económica de la ciudad de Cartago, a causa de los efectos de una avalancha que transite por el río Reventado, Costa Rica. Revista Geológica América Central, 15, 65-80.

Parsons, W. (1967). Irazú volcano. Newsletter, Cranbrook Institute of Science, 36(6), 70-75.

Young, S. R., Baxter, P. J., Pomonis, A., Ernst, G. G. J. y Benson, C. (1998). Volcanic hazards and community preparedness at volcán Irazú, Costa Rica. British Geological Survey Technical Report, WC/98/16R, 1-20. 


\section{ANEXO 1}

Cuadro 10

Pérdidas en cultivos por la actividad del Irazú (1963-1965) en valor presente al 2016.

\begin{tabular}{|c|c|c|c|c|}
\hline Café & Tabaco & Papa & Varios cultivos & Referencia \\
\hline$\not{C} 53374568662$ & $\mathbb{C} 413267393$ & $\not \subset 521202112$ & - & GCROP (1964) \\
\hline 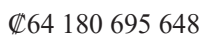 & $\mathbb{C} 425664157$ & $\not \subset 520256192$ & - & ICE (1965) \\
\hline$\not \mathbb{4} 641631150$ & - & - & - & MAG (1963) \\
\hline$\not \mathbb{1} 12580740636$ & - & - & $\not \subset 629037032$ & Ambrister (1964) \\
\hline $\mathbb{C} 12580740636$ & - & - & - & Parsons (1967) \\
\hline
\end{tabular}

\section{ANEXO 2}

Cuadro 11

Pérdidas en ganadería por la actividad del Irazú (1963-1965) en valor presente al 2016

\begin{tabular}{ccc}
\hline Ganado sacrificado por & Total & Referencia \\
\hline Afectación por ceniza & $\mathbb{C} 638496235$ & GCROP (1964) \\
Menor producción lechera & $\mathbb{C} 454609320$ & GCROP (1964) \\
Improductividad & $\mathbb{C} 411371773$ & MAG (1963) \\
No especificado & $\mathbb{C} 66214244$ & ICE (1965) \\
\hline
\end{tabular}

\section{ANEXO 3}

Cuadro 12

Pérdidas indirectas por la actividad del Irazú (1963-1965) en valor presente al 2016.

\begin{tabular}{|c|c|}
\hline Pérdidas & Total \\
\hline Devaluación de tierras & $\not 147296017427$ \\
\hline Servicios Públicos & $\not \subset 1891840697$ \\
\hline
\end{tabular}




\section{ANEXO 4}

Cuadro 13

Inversiones para la recuperación por la actividad del Irazú (1963 - 1965) en valor presente al 2016.

\begin{tabular}{|c|c|}
\hline Programa & Costo \\
\hline Pastos & $\not \subset 6124834257$ \\
\hline Atención al ganado & $\mathbb{C 2} 581220660$ \\
\hline Combate plagas del café & $\mathbb{E} 2364800871$ \\
\hline Diques y dragados & $\not 46541509805$ \\
\hline Infraestructura vial & $\mathbb{C} 4034350286$ \\
\hline Limpieza de calles & $\mathbb{C} 860265842$ \\
\hline Agua potable & $\mathbb{C} 976189800$ \\
\hline Vivienda & $\mathbb{C} 6772553215$ \\
\hline Financiamiento a cafetaleros & $\mathbb{C} 9459203485$ \\
\hline Industrias en Taras & $\not 2364800871$ \\
\hline Carreteras & $\mathbb{C} 3987054260$ \\
\hline Hospital San Juan de Dios debido a la ceniza & $\mathbb{C} 505793070$ \\
\hline Total & $\not 446572576433$ \\
\hline
\end{tabular}

